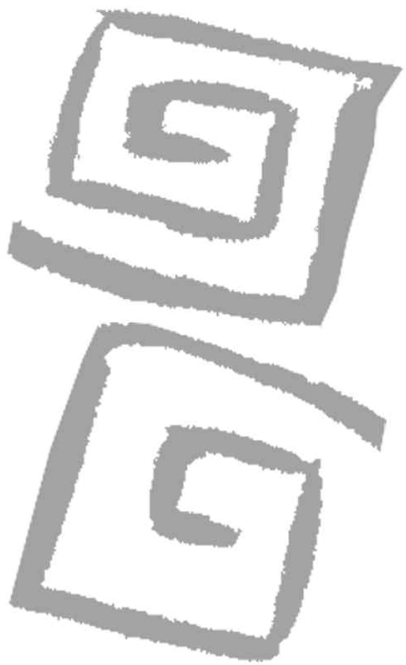

\title{
Tuberculosis en Argentina: desigualdad social y de género
}

\author{
Tuberculosis in Argentina: \\ social and gender inequality
}

Bossio, Juan Carlos ${ }^{1}$; Arias, Sergio Javier²; Fernández, Hugo Roberto ${ }^{3}$

\footnotetext{
${ }^{1}$ Bioquímico. Diplomado en Salud Pública. Jefe de Departamento Programas de Salud, Instituto Nacional de Enfermedades Respiratorias "Emilio Coni", Administración Nacional de Laboratorios e Institutos de Salud "Carlos G. Malbrán", Argentina. jcbossio@arnet.com.ar

2 Bioquímico. Magister en Epidemiología. Profesional del Departamento Programas de Salud, Instituto Nacional de Enfermedades Respiratorias "Emilio Coni", Administración Nacional de Laboratorios e Institutos de Salud "Carlos G. Malbrán", Argentina. sergio_arias@arnet.com.ar

${ }^{3}$ Bioquímico. Jefe de la División Epidemiología, Departamento Programas de Salud, Instituto Nacional de Enfermedades Respiratorias "Emilio Coni", Administración Nacional dé Laboratorios e Institutos de Salud "Carlos G. Malbrán", Argentina. fernanhg@msn.com
}

RESUMEN Este artículo se propone describir la distribución de la tuberculosis en Argentina y analizar la desigualdad social y de género de la distribución en el período 1990-2011. Para ello, se emplearon las cifras oficiales de notificación de casos de tuberculosis del país, se calcularon tasas por 100.000 habitantes por jurisdicción y departamentos, por grupos de edad y por sexo. La desigualdad en la distribución se analizó mediante el índice de Gini y su asociación con las condiciones sociales mediante el índice de concentración y el índice de efecto; la tendencia se estudió mediante regresión exponencial. Los resultados muestran que las tasas de tuberculosis son mayores entre los 20 y 34 años de edad y son superiores en varones. Existe una marcada desigualdad entre jurisdicciones (índice de Gini $=0,273$ ) y es mayor por departamentos (índice de Gini = 0,391), superior en mujeres $(0,411)$ que en varones $(0,394)$. La distribución de la tuberculosis se asoció a peores condiciones sociales (índice de efecto $=-0,212$ ), y esta asociación fue mayor en las mujeres (índice de efecto $=11,4 ; 6,4 ; 16,4$ ). La tendencia del problema al descenso entre 1990 y 2011 no ha contribuido a reducir las brechas entre jurisdicciones y departamentos ni tampoco a reducir la desigualdad en la distribución de la tuberculosis asociada a las desfavorables condiciones sociales de la población.

PALABRAS CLAVE Tuberculosis; Desigualdad en Salud; Desigualdad Social; Argentina.

ABSTRACT This article seeks to describe the distribution of tuberculosis in Argentina and analyze the social and gender inequality of the distribution within the period of 1990-2011. In order to accomplish this, the official data from tuberculosis case notifications in the country was used, and the rates were calculated per 100,000 inhabitants by jurisdiction and department, by age group and by sex. The inequality in the distribution was analyzed using the Gini index and the association with social conditions using the concentration index and the effect index; the trend was studied using exponential regression. The results demonstrate that tuberculosis rates are higher between 20 and 34 years of age and are greater in males. There is a marked inequality among jurisdictions (Gini index $=0.273$ ) which is even greater among departments (Gini index $=0.391$ ) and is higher in females (0.411) than in males (0.394). The distribution of tuberculosis was associated with worse social conditions (effect index $=-0.212$ ) and this association was stronger in females (effect index = $11.4,6.4,16.4)$. The downward trend in tuberculosis rates observed between 1990 and 2011 has not contributed to reducing the gaps among jurisdictions and departments nor has it reduced the inequality in the distribution of tuberculosis associated with the unfavorable social conditions of the population.

KEY WORDS Tuberculosis; Health Inequality; Social Inequality; Argentina. 


\section{INTRODUCCIÓN}

La tuberculosis es considerada en la actualidad una de las enfermedades infecciosas de mayor importancia global, tanto en términos del número de casos nuevos anuales que produce, como de la persistencia de un número importante de defunciones por esta causa que siguen ocurriendo a pesar de contarse desde hace decenios con tecnologías de diagnóstico y tratamiento efectivos $(1,2)$. Aunque la aparición de la infección por el virus de la inmunodeficiencia humana (VIH) y el surgimiento de bacterias resistentes y multirresistentes a los medicamentos disponibles para el tratamiento de la tuberculosis han representado un importante desafío para el control de la enfermedad, estas condiciones no alcanzan por sí solas para explicar la continua ocurrencia de casos y muertes por tuberculosis a nivel mundial (3-5). Estas situaciones han contribuido más bien a agravar la situación existente, caracterizada por una gran desigualdad en la distribución de los casos de tuberculosis entre los países y hacia el interior de cada uno de ellos (6).

Múltiples razones pueden encontrarse para justificar que, aun disponiendo de los medios para el diagnóstico y el tratamiento de la mayoría de los casos de tuberculosis, esta enfermedad continúe constituyendo una prioridad en la mayoría de los países en desarrollo (2). Las deficientes condiciones de vida de la población, que repercuten en su condición nutricional, en la probabilidad de infectarse con el bacilo de la tuberculosis y en la capacidad del organismo de responder a la infección son en gran parte responsables de la mayor ocurrencia de tuberculosis en muchas áreas geográficas y grupos de población que se suman a los factores biológicos que incrementan la vulnerabilidad del organismo a la infección y la enfermedad (7-9).

En condiciones sociales desfavorables y con escasa o deficiente oferta de atención, los casos de tuberculosis pueden permanecer un tiempo variable antes de ser correctamente diagnosticados y de iniciar el tratamiento de la enfermedad (2). Durante este lapso, los casos de tuberculosis constituyen fuentes de diseminación de la enfermedad entre la población sana, y aquellas personas que compartan más tiempo con los enfermos no detectados y sin tratamiento tendrán más riesgo de infectarse $y$, potencialmente, de enfermar $(5,10,11)$.

La presencia del bacilo de la tuberculosis es una condición necesaria pero no suficiente para que ocurra la infección en una persona. Otros determinantes, como la condición nutricional e inmunitaria, las condiciones de hacinamiento, la escasa ventilación y la escasa higiene, entre otros, contribuyen a aumentar la probabilidad de que, en presencia del bacilo, ocurra la infección y, posteriormente, la enfermedad $(12,13)$.

En la República Argentina, la tuberculosis constituye un problema de salud pública prioritario desde hace muchos años y en todas las jurisdicciones del país se realizan actividades de búsqueda y detección de casos y de tratamiento gratuito de los enfermos por parte de los programas de control (14).

Si bien se ha registrado un importante descenso en el número de casos anuales notificados, la tuberculosis todavía continúa afectando a un número considerable de personas, y la tendencia registrada en los últimos decenios no ha mostrado un ritmo de reducción compatible con el esperado, en función de la implementación de las acciones de búsqueda y detección de casos y de tratamiento de los enfermos. Además, el ritmo de reducción de la tuberculosis pulmonar confirmada por examen directo de la expectoración (que constituye la forma de la enfermedad que disemina el bacilo de la tuberculosis poniendo en riesgo de infectarse a la población sana), ha sido menor al que ha registrado el total de casos de la enfermedad $(15,16)$.

El análisis de la distribución desigual de la tuberculosis en el país, así como de la asociación entre esta distribución y los determinantes sociales resulta de interés para poder identificar las áreas y grupos de población que, en la actualidad, no solo concentran la mayor cantidad de casos de tuberculosis sino que están expuestos a un mayor riesgo de contraer la enfermedad en el futuro, a partir del mayor número de casos que diseminan la enfermedad en la población. 


\section{METODOLOGÍA}

Los datos de notificación de casos de tuberculosis se obtuvieron de las cifras oficiales del Instituto Nacional de Enfermedades Respiratorias (INER) "Emilio Coni", a partir de los datos del Programa de Control de la Tuberculosis de cada una de las 24 jurisdicciones del país. Se analizaron todos los casos de tuberculosis notificados (todas las formas), desagregados por edad, sexo y residencia, y los casos de tuberculosis de localización pulmonar confirmados por examen directo (examen directo positivo).

Los datos de población, para el cálculo de las tasas de notificación por cada 100.000 habitantes, se obtuvieron de las estimaciones y proyecciones del Instituto Nacional de Estadística y Censos (INDEC) basadas en los censos nacionales de 1980, 1991, 2001 y 2010, para el total del país, por jurisdicción (provincias y Ciudad Autónoma de Buenos Aires) y por departamento (o partido, en el caso de la provincia de Buenos Aires), y por sexo. Para el año 2011 se utilizaron proyecciones basadas en la variación intercensal 1991-2001, aplicada a la población de 2010.

Los datos de condición social de la población se obtuvieron de las cifras publicadas por el INDEC correspondientes al Censo Nacional de Población 2010. Se seleccionaron como indicadores sociales la población de ambos sexos en viviendas precarias (considerando como tal la población en rancho, casilla, pieza/s en inquilinato, pieza/s en hotel o pensión o local no construido para vivienda) y la población de diez años o más analfabeta, desagregada en varones y mujeres; ambos indicadores disponibles por jurisdicción (provincias y Ciudad Autónoma de Buenos Aires) y por departamento o partido.

Para el análisis de las diferencias en la notificación de casos según grupos de edad, sexo y residencia (jurisdicción y departamento/partido) se calcularon tasas específicas por 100.000 habitantes. Las tasas se compararon mediante el rango, la razón y la diferencia porcentual entre tasas.

El análisis de la desigualdad en la distribución de la tuberculosis por residencia se realizó mediante el índice de Gini, ordenando las jurisdicciones o departamentos/partidos según su tasa de notificación de tuberculosis. El índice de Gini se calculó para los casos de tuberculosis (todas las formas) de todas las edades y de 20 a 34 años, según sexo y según clasificación del caso de tuberculosis (todas las formas y pulmonares con examen directo positivo).

El análisis de la desigualdad en la distribución de la tuberculosis por residencia y su asociación con las condiciones sociales se realizó mediante el cálculo del índice de concentración, ordenando las jurisdicciones o departamentos/partidos en función de la proporción de población en viviendas precarias (para ambos sexos) y de la proporción de población de diez años o más analfabeta (para ambos sexos, para varones y para mujeres). También se empleó el índice de efecto, calculando el coeficiente de regresión de la tasa de notificación en función de las variables sociales mencionadas. Se utilizó el coeficiente de regresión lineal de Pearson con su intervalo de confianza del 95\%.

El análisis de la tendencia del problema se realizó mediante regresión de la tasa de notificación de casos en función del tiempo, considerando un modelo exponencial. Se seleccionó como año de inicio 1990, teniendo en cuenta que es el año base para los Objetivos de Desarrollo del Milenio (ODM) referidos a tuberculosis y establecidos por la Organización de las Naciones Unidas en el año 2000. Se analizó el período completo (1990-2011) y dos subperíodos (1990-2000 y 2000-2011), para comparar la tendencia a partir de la adopción de los ODM.

Se calculó el coeficiente de regresión lineal del logaritmo natural de base y de la tasa de notificación y se expresó como variación porcentual anual promedio, con su intervalo de confianza del $95 \%$.

La tendencia de la desigualdad en la distribución de los casos de tuberculosis por jurisdicción y de la desigualdad asociada a las condiciones sociales, se realizó mediante el cálculo del índice de Gini y del índice de concentración para los años 1990, 2000 y 2011, considerando el año de inicio y final de la serie temporal analizada y el año 2000 en el que se fijaron los ODM.

Se utilizaron los programas Epi Info Versión 3.5.3 y Excel 2010. La investigación respetó la Ley de Secreto Estadístico preservando la confidencialidad de las personas involucradas. 


\section{RESULTADOS}

\section{Carga de enfermedad}

En la República Argentina se notifican anualmente entre 9.000 y 10.000 casos nuevos de tuberculosis. En 2011, según la última información oficial disponible, 9.657 casos nuevos fueron notificados por las 24 jurisdicciones, lo que representó una tasa de 23,6 casos por cada 100.000 habitantes. El número de casos notificados en 2011 fue 2,8\% superior al del año anterior (9.393), aunque la tasa de notificación tuvo un aumento menor (1,7\% más alta que la de 2010) por el mayor incremento porcentual del número de habitantes del país.

Aunque todos los casos de tuberculosis representan la carga total de la enfermedad en la población, los casos de localización pulmonar confirmados por examen directo (es decir, por identificación del bacilo tuberculoso en muestras de expectoración de los pacientes) resultan más relevantes por su implicancia en la transmisión de la enfermedad, ya que son las fuentes de contagio para la población sana. Más de la mitad de los nuevos casos de tuberculosis notificados en 2011 $(55,3 \%)$ fueron de localización pulmonar y confirmados por examen directo (5.338 casos), lo que representó 13,1 casos por cada 100.000 habitantes. El número de casos bacilíferos notificado en 2011 también fue un 2,2\% superior al de 2010 .

\section{Desigualdad por edad y sexo}

El análisis de la tuberculosis por grupos de edades muestra que Argentina presenta una concentración de casos en la población de adultos jóvenes. En 2010-2011, del total de casos de tuberculosis notificados, el 35,8\% tenía entre 20 y 34 años (Cuadro 1). La tasa de tuberculosis de este grupo de edad fue 34,7 por 100.000 habitantes, la mayor tasa de tuberculosis por habitantes en la República Argentina y un 76,1\% más alta que la del resto de la población (19,7 por 100.000 habitantes). La proporción de los casos de tuberculosis bacilíferos de 20 a 34 años es mayor que la de todos los casos: en 2011, el $60,3 \%$ de los casos bacilíferos pertenecía a ese grupo de edad.

La tuberculosis se presenta con más frecuencia entre hombres que entre mujeres. El $57,9 \%$ de los casos de tuberculosis del bienio 2010-2011 ocurrió en hombres, mientras que hubo un $42,1 \%$ de notificaciones de sexo femenino. Esta diferencia en la cantidad de casos por sexo se traduce también en una mayor tasa de notificación entre hombres que entre mujeres (Cuadro 1).

A su vez, tanto en hombres como en mujeres, la notificación de casos es también mayor entre los 20 y 34 años. Sin embargo, la distribución de la tuberculosis por edad es diferente en los varones que en las mujeres y se puede observar que las tasas de notificación de

Cuadrol. Notificación de casos de tuberculosis (todas las formas) según sexo y grupos de edad. Números, tasas (por 100.000 habitantes) y porcentajes. Argentina, 2010-2011.

\begin{tabular}{|c|c|c|c|c|c|c|c|c|c|}
\hline \multirow{2}{*}{$\begin{array}{l}\text { Grupos de edad } \\
\text { (en años) }\end{array}$} & \multicolumn{3}{|c|}{ Mujeres } & \multicolumn{3}{|c|}{ Varones } & \multicolumn{3}{|c|}{ Total } \\
\hline & No. & Tasa & $\%$ & No. & Tasa & $\%$ & No. & Tasa & $\%$ \\
\hline 0 a 19 & 1.669 & 12,5 & 20,8 & 1.952 & 14,1 & 17,7 & 3.621 & 13,3 & 19,0 \\
\hline 20 a 34 & 2.992 & 30,7 & 37,3 & 3.833 & 38,7 & 34,8 & 6.827 & 34,7 & 35,8 \\
\hline 35 a 44 & 1.099 & 21,2 & 13,7 & 1.527 & 29,7 & 13,9 & 2.628 & 25,4 & 13,8 \\
\hline 45 a 64 & 1.474 & 18,0 & 18,4 & 2.609 & 34,5 & 23,7 & 4.083 & 25,9 & 21,4 \\
\hline 65 y más & 780 & 15,5 & 9,7 & 1.070 & 31,1 & 9,7 & 1.852 & 21,8 & 9,7 \\
\hline Sin especificar & 11 & - & 0,1 & 28 & - & 0,3 & 39 & - & 0,2 \\
\hline Total & 8.025 & 19,3 & 100,0 & 11.019 & 27,6 & 11,0 & 19.050 & 23,4 & 100,0 \\
\hline
\end{tabular}

Fuente: Elaboración propia a partir de información oficial de los programas de control de la tuberculosis de las 24 jurisdicciones del país, consolidada por el Instituto Nacional de Enfermedades Respiratorias (INER) "Emilio Coni", Administración Nacional de Laboratorios e Institutos de Salud (ANLIS), del Ministerio de Salud de la Nación, Argentina, 2012. 
Cuadro 2. Notificación de casos de tuberculosis (todas las formas) según sexo y grupos de edad. Tasas (por 100.000 habitantes) y razón de tasas entre hombres y mujeres. Argentina, 2010-2011.

\begin{tabular}{|c|c|c|c|}
\hline \multirow{2}{*}{$\begin{array}{l}\text { Grupos de edad } \\
\text { (en años) }\end{array}$} & \multicolumn{2}{|c|}{ Tasa } & \multirow{2}{*}{$\begin{array}{l}\text { Razón de tasas } \\
\text { (hombre-mujer) }\end{array}$} \\
\hline & Mujeres & Varones & \\
\hline 0 a 19 & 12,5 & 14,1 & 1,1 \\
\hline 20 a 34 & 30,7 & 38,7 & 1,3 \\
\hline 35 a 44 & 21,2 & 29,7 & 1,4 \\
\hline 45 a 64 & 18,0 & 34,5 & 1,9 \\
\hline 65 y más & 15,5 & 31,1 & 2,0 \\
\hline Total & 19,3 & 27,6 & 1,4 \\
\hline \multicolumn{4}{|c|}{$\begin{array}{l}\text { Fuente: Elaboración propia a partir de información oficial de los programas } \\
\text { de control de la tuberculosis de las } 24 \text { jurisdicciones del país, consolidada } \\
\text { por el Instituto Nacional de Enfermedades Respiratorias (INER) "Emilio Coni" } \\
\text { Administración Nacional de Laboratorios e Institutos de Salud (ANLIS), de } \\
\text { Ministerio de Salud de la Nación, Argentina, } 2012 \text {. }\end{array}$} \\
\hline
\end{tabular}

tuberculosis de mujeres de 20 a 34 años son $70 \%$ más altas comparadas con las de 45 a 64 años, mientras que en los varones son solo un $12 \%$ más altas en los jóvenes que en los mayores. La diferencia en el peso de la tuberculosis en el grupo de 20 a 34 años en las mujeres se hace aun mayor en los casos bacilíferos: las mujeres de 20 a 34 años registran una tasa $94 \%$ más alta que las de 45 a 64 años mientras que, en los hombres, los jóvenes tienen una tasa solo $5 \%$ más alta que los adultos de 45 a 64 años.

Dada la diferencia en el comportamiento por edad en cada género, la razón entre las tasas de la enfermedad en varones y en mujeres se reduce en la población joven y aumenta con la edad. Mientras que entre los 45 y los 64 años la tasa de tuberculosis en varones es casi el doble que en mujeres, entre los 20 y los 34 años la tasa de varones es solamente $26 \%$ superior a la de mujeres (Cuadro 2).

\section{Desigualdad por residencia}

La distribución de la tuberculosis no es uniforme en el país, se observan diferencias importantes entre jurisdicciones, que son mayores aun entre partidos (en la provincia de Buenos Aires) y departamentos (en el resto de las provincias).

\section{Diferencias por jurisdicción}

Las dos terceras partes de los casos de tuberculosis que se notifican en Argentina residen en las cuatro jurisdicciones más grandes del país, con más de tres millones de habitantes: Provincia de Buenos Aires, Ciudad Autónoma de Buenos Aires, Córdoba y Santa Fe. Sin embargo,

Cuadro 3. Notificación de casos de tuberculosis (todas las formas) por jurisdicciones. Números, tasas (por 100.000 habitantes) porcentajes y razón de tasas (tomando como referencia el valor nacional). Argentina, 2011.

\begin{tabular}{|c|c|c|c|c|}
\hline \multirow[t]{2}{*}{ Jurisdicción } & \multicolumn{4}{|c|}{ Tuberculosis (todas las formas) } \\
\hline & No. & Tasa & $\%$ & Razón de tasas \\
\hline Buenos Aires & 4.433 & 28,7 & 45,9 & 1,2 \\
\hline CABA & 1.210 & 39,5 & 12,5 & 1,7 \\
\hline Catamarca & 36 & 8,7 & 0,4 & 0,4 \\
\hline Córdoba & 339 & 9,9 & 3,5 & 0,4 \\
\hline Corrientes & 313 & 29,0 & 3,2 & 1,2 \\
\hline Chaco & 83 & 17,5 & 0,9 & 0,7 \\
\hline Chubut & 247 & 23,6 & 2,6 & 1,0 \\
\hline Entre Ríos & 210 & 16,2 & 2,2 & 0,7 \\
\hline Formosa & 211 & 37,4 & 2,2 & 1,6 \\
\hline Jujuy & 413 & 58,4 & 4,3 & 2,5 \\
\hline La Pampa & 36 & 10,4 & 0,4 & 0,4 \\
\hline La Rioja & 20 & 5,5 & 0,2 & 0,2 \\
\hline Mendoza & 127 & 7,1 & 1,3 & 0,3 \\
\hline Misiones & 179 & 15,9 & 1,9 & 0,7 \\
\hline Neuquén & 26 & 4,5 & 0,3 & 0,2 \\
\hline Río Negro & 42 & 6,9 & 0,4 & 0,3 \\
\hline Salta & 658 & 51,0 & 6,8 & 2,2 \\
\hline San Juan & 80 & 11,0 & 0,8 & 0,5 \\
\hline San Luis & 36 & 7,7 & 0,4 & 0,3 \\
\hline Santa Cruz & 58 & 24,4 & 0,6 & 1,0 \\
\hline Santa Fe & 514 & 15,6 & 5,3 & 0,7 \\
\hline Santiago del Estero & 126 & 14,1 & 1,3 & 0,6 \\
\hline Tierra del Fuego & 19 & 23,3 & 0,2 & 0,6 \\
\hline Tucumán & 207 & 13,5 & 2,1 & 0,6 \\
\hline Sin especificar & 34 & - & 0,4 & - \\
\hline Total país & 9.657 & 23,6 & 100,0 & 1,0 \\
\hline
\end{tabular}

Fuente: Elaboración propia a partir de información oficial de los programas de control de la tuberculosis de las 24 jurisdicciones del país, consolidada por el Instituto Nacional de Enfermedades Respiratorias (INER) "Emilio Coni", Administración Nacional de Laboratorios e Institutos de Salud (ANLIS), del Ministerio de Salud de la Nación, Argentina, 2012. 
la tasa de notificación de estas jurisdicciones es distinta entre sí, ya que mientras la Ciudad Autónoma de Buenos Aires y la Provincia de Buenos Aires registran tasas más altas que el promedio nacional (67\% y $22 \%$, respectivamente), las provincias de Córdoba y Santa Fe tienen tasas por debajo del promedio nacional $(58 \%$ y $34 \%$ más bajas, respectivamente).

El rango de las tasas de notificación de casos de tuberculosis (todas las formas) de cualquier edad va de 58,4 por 100.000 en la provincia de Jujuy a 4,5 por 100.000 en la provincia de Neuquén, lo que indica una diferencia de 13 veces entre la menor y la mayor de las tasas provinciales en 2011. A su vez, la provincia de Jujuy presenta en 2011 una tasa 2,4 veces más alta que la tasa de Argentina para el mismo año (Cuadro3, Figura 1).

La desigualdad en la distribución de la tuberculosis en el país se refleja en el valor del índice de Gini que, para el bienio 2010-2011 fue 0,273 . En este bienio, las dos provincias con mayor tasa de notificación, que concentran el 5\% de la población, notificaron el $11 \%$ de los casos de tuberculosis del país, mientras que las tres provincias con menor tasa, que concentran el $4 \%$ de la población, aportaron solamente el $1 \%$ de los casos de tuberculosis del país. La desigualdad entre jurisdicciones fue similar en varones y en mujeres (el índice de Gini fue 0,277 en varones y 0,278 en mujeres).

En los jóvenes de 20 a 34 años la desigualdad en la distribución y las brechas entre jurisdicciones son mayores. El índice de Gini para ambos sexos del grupo de 20 a 34 años fue 0,317, para el bienio 2010-2011 y, también en este caso, similar en varones y en mujeres: 0,322 y 0,315 respectivamente. El índice de Gini de 20 a 34 años es $16,1 \%$ más alto que para todas las edades.

Además de este aumento en la desigualdad en la distribución de la tuberculosis, en la población joven también cambia el ordenamiento respecto de las provincias con mayor a menor

Figura 1. Notificación de casos de tuberculosis (todas las formas) por jurisdicciones. Tasas (por 100.000 habitantes). Argentina, 2010-2011.

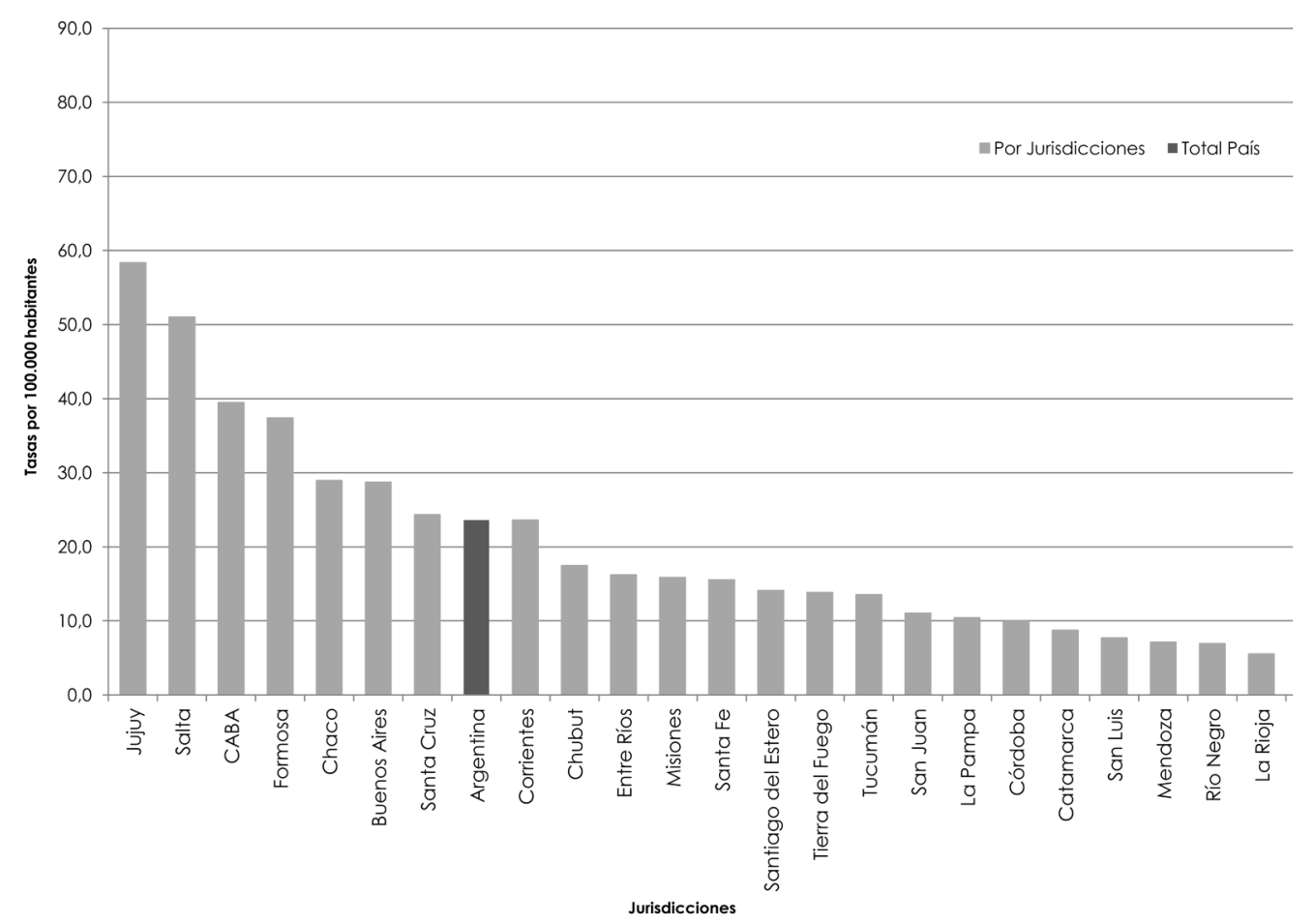

Fuente: Elaboración propia a partir de información oficial de los programas de control de la tuberculosis de las 24 jurisdicciones del país, consolidada por el Instituto Nacional de Enfermedades Respiratorias (INER) "Emilio Coni", Administración Nacional de Laboratorios e Institutos de Salud (ANLIS), del Ministerio de Salud de la Nación, Argentina, 2012. 
tasa de notificación. En los jóvenes, la Ciudad Autónoma de Buenos Aires pasa a ocupar el primer lugar con la tasa más alta de notificación de casos de tuberculosis (todas las formas) de 20 a 34 años del país: 82,8 por 100.000, cifra 13,9 veces más alta que la de la provincia de La Rioja, que notificó en ese bienio la tasa más baja en este grupo de edad (Cuadro 4, Figura 2). La Ciudad Autónoma de Buenos Aires, que aporta el $7 \%$ de la población de 20 a 34 años del país, notificó el $17 \%$ de todos los casos de tuberculosis de ese grupo de edad en el bienio 2010-2011.

Considerando solamente los casos de tuberculosis en jóvenes de 20 a 34 años que tuvieron localización pulmonar y se confirmaron por examen directo, las brechas entre jurisdicciones son similares y las dos con mayor tasa (Ciudad Autónoma de Buenos Aires y Formosa, ambas con una tasa de 45,98 por 100.000 habitantes) aportan el $8 \%$ de la población de esa edad y el $18 \%$ de los casos de tuberculosis del país.
Las cinco jurisdicciones con menor tasa que aportan una proporción similar de población, notificaron solamente el $1 \%$ de los casos de tuberculosis en jóvenes. El índice de Gini para la distribución de estos casos por jurisdicción también fue ligeramente superior en mujeres que en varones: 0,308 y 0,291, respectivamente.

\section{Diferencias por departamento}

Las brechas en la distribución de la tuberculosis en el país entre departamentos son mayores y permiten identificar algunos con tasas de notificación mucho más elevadas que las provincias: dos departamentos de la provincia de Formosa (Ramón Lista y Matacos) tuvieron tasas de notificación de casos de tuberculosis (todas las formas) superiores a 200 por 100.000 habitantes (250,1 y 241,7, respectivamente) mientras que 84 departamentos del país no notificaron ningún caso de tuberculosis durante el bienio 2010-

Figura 2. Notificación de casos de tuberculosis (todas las formas) entre 20 y 34 años por jurisdicciones. Tasas (por 100.000 habitantes). Argentina, 2010-2011.

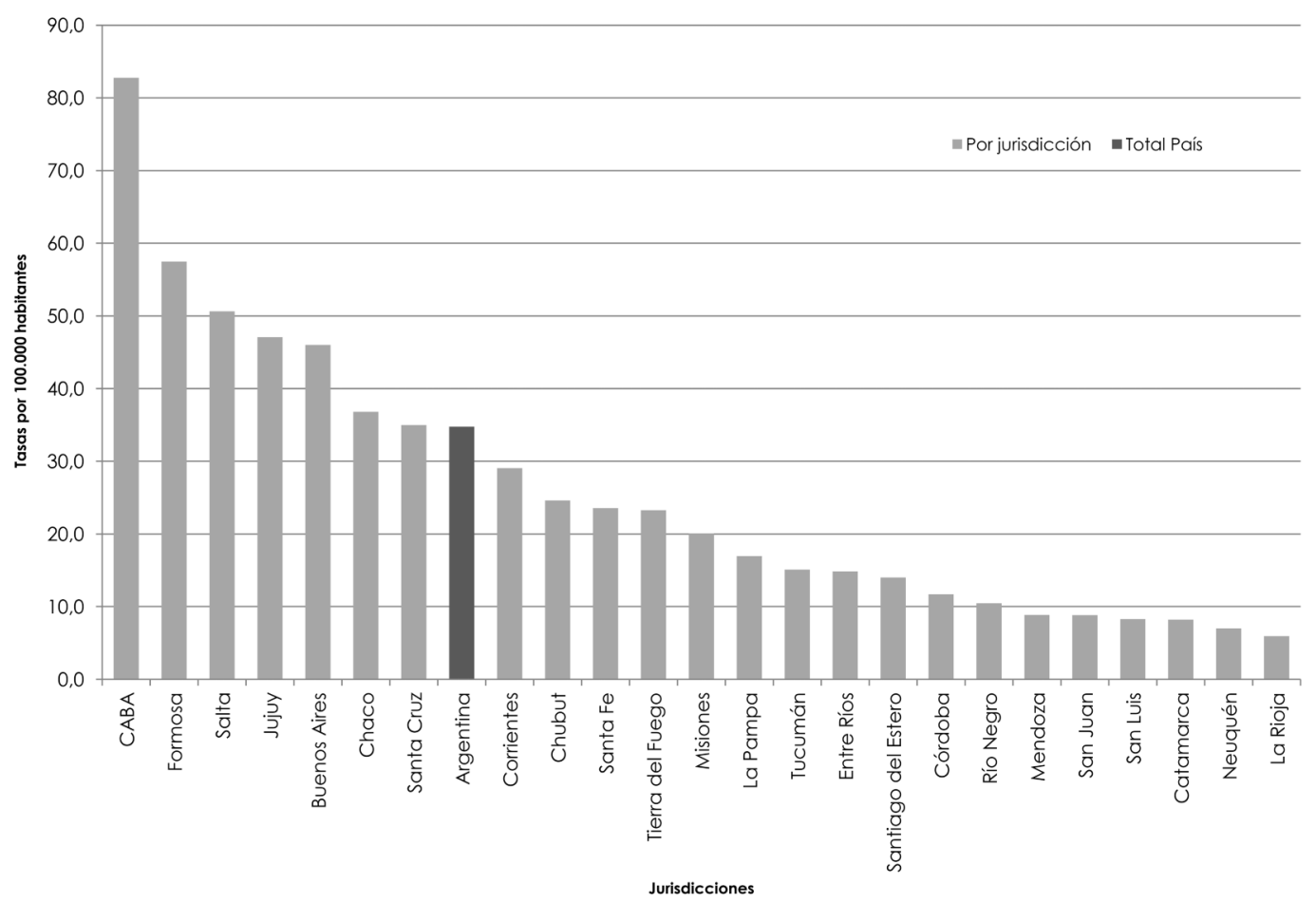

Fuente: Elaboración propia a partir de información oficial de los programas de control de la tuberculosis de las 24 jurisdicciones del país, consolidada por el Instituto Nacional de Enfermedades Respiratorias (INER) "Emilio Coni", Administración Nacional de Laboratorios e Institutos de Salud (ANLIS), del Ministerio de Salud de la Nación, Argentina, 2012. 
Cuadro 4. Notificación de casos de tuberculosis (todas las formas y pulmonares examen directo positivo) entre 20 y 34 años por jurisdicciones. Números, tasas (por 100.000 habitantes) y porcentajes. Argentina, 2010-2011.

\begin{tabular}{|c|c|c|c|c|c|c|}
\hline \multirow[t]{2}{*}{ Jurisdicción } & \multicolumn{3}{|c|}{$\begin{array}{c}\text { Tuberculosis } \\
\text { (todas las formas) }\end{array}$} & \multicolumn{3}{|c|}{$\begin{array}{c}\text { Tuberculosis (pulmonares examen } \\
\text { directo positivo) }\end{array}$} \\
\hline & No. & Tasa & $\%$ & No. & Tasa & $\%$ \\
\hline Buenos Aires & 3.391 & 46,0 & 49,7 & 1.994 & 27,1 & 47,8 \\
\hline Ciudad Autónoma de Buenos Aires & 1.132 & 82,7 & 16,6 & 629 & 46,0 & 15,1 \\
\hline Catamarca & 16 & 8,2 & 0,2 & 11 & 5,6 & 0,3 \\
\hline Córdoba & 198 & 36,8 & 2,9 & 136 & 25,3 & 3,3 \\
\hline Corrientes & 57 & 24,6 & 0,8 & 31 & 13,4 & 0,7 \\
\hline Chaco & 199 & 11,7 & 2,9 & 141 & 8,3 & 3,4 \\
\hline Chubut & 150 & 29,1 & 2,2 & 118 & 22,9 & 2,8 \\
\hline Entre Ríos & 89 & 14,9 & 1,3 & 64 & 10,7 & 1,5 \\
\hline Formosa & 155 & 57,5 & 2,3 & 124 & 46,0 & 3,0 \\
\hline Jujuy & 164 & 47,1 & 2,4 & 99 & 28,4 & 2,4 \\
\hline La Pampa & 27 & 16,9 & 0,4 & 19 & 11,9 & 0,5 \\
\hline La Rioja & 11 & 5,9 & 0,2 & 7 & 3,8 & 0,2 \\
\hline Mendoza & 76 & 8,8 & 1,1 & 57 & 6,6 & 1,4 \\
\hline Misiones & 107 & 20,1 & 1,6 & 82 & 15,4 & 2,0 \\
\hline Neuquén & 20 & 7,0 & 0,3 & 8 & 2,8 & 0,2 \\
\hline Río Negro & 30 & 10,5 & 0,4 & 20 & 7,0 & 0,5 \\
\hline Salta & 316 & 50,6 & 4,6 & 174 & 27,9 & 4,2 \\
\hline San Juan & 30 & 8,8 & 0,4 & 13 & 3,8 & 0,3 \\
\hline San Luis & 18 & 8,3 & 0,3 & 12 & 5,5 & 0,3 \\
\hline Santa Cruz & 39 & 35,0 & 0,6 & 22 & 19,7 & 0,5 \\
\hline Santa Fe & 383 & 23,5 & 5,6 & 278 & 17,1 & 6,7 \\
\hline Santiago del Estero & 63 & 14,0 & 0,9 & 22 & 4,9 & 0,5 \\
\hline Tierra del Fuego & 15 & 23,3 & 0,2 & 10 & 15,5 & 0,2 \\
\hline Tucumán & 117 & 15,1 & 1,7 & 80 & 10,3 & 1,9 \\
\hline Sin especificar & 9 & - & 0,1 & 9 & - & 0,2 \\
\hline Total país & 6.827 & 34,7 & 100,0 & 4.170 & 21,2 & 100,0 \\
\hline
\end{tabular}

Fuente: Elaboración propia a partir de información oficial de los programas de control de la tuberculosis de las 24 jurisdicciones del país, consolidada por el Instituto Nacional de Enfermedades Respiratorias (INER) "Emilio Coni", Administración Nacional de Laboratorios e Institutos de Salud (ANLIS), del Ministerio de Salud de la Nación, Argentina, 2012.

2011 ; y 33 departamentos tuvieron tasas inferiores a 4,5 por 100.000 , cifras más de 50 veces por debajo de las que se registraron en los dos departamentos de Formosa (Figura 3). Como comparación, se puede mencionar que según el último informe de la Organización Mundial de la Salud sobre la situación de la tuberculosis en el mundo, las tasas informadas en Matacos y Ramón Lista en Formosa son similares a las de países como Bangladesh (225 por 100.000), Etiopía (261 por
100.000) o Pakistán (231 por 100.000), que se encuentran entre los países con más alta carga de esta enfermedad en el mundo.

La desigualdad entre departamentos, medida a través del índice de Gini, fue superior a la desigualdad entre provincias, con un valor del índice de Gini de 0,391. Los 29 departamentos que concentran el $10 \%$ de la población del país aportaron el 25,7\% de todos los casos de tuberculosis que se notificaron el último bienio 2010- 
Figura 3. Notificación de casos de tuberculosis (todas las formas) en departamentos seleccionados (>p95 y < 5 , calculados para los 426 departamentos/partidos con por lo menos un caso notificado en el bienio). Tasas por 100.000 habitantes. Argentina, 2010-2011.

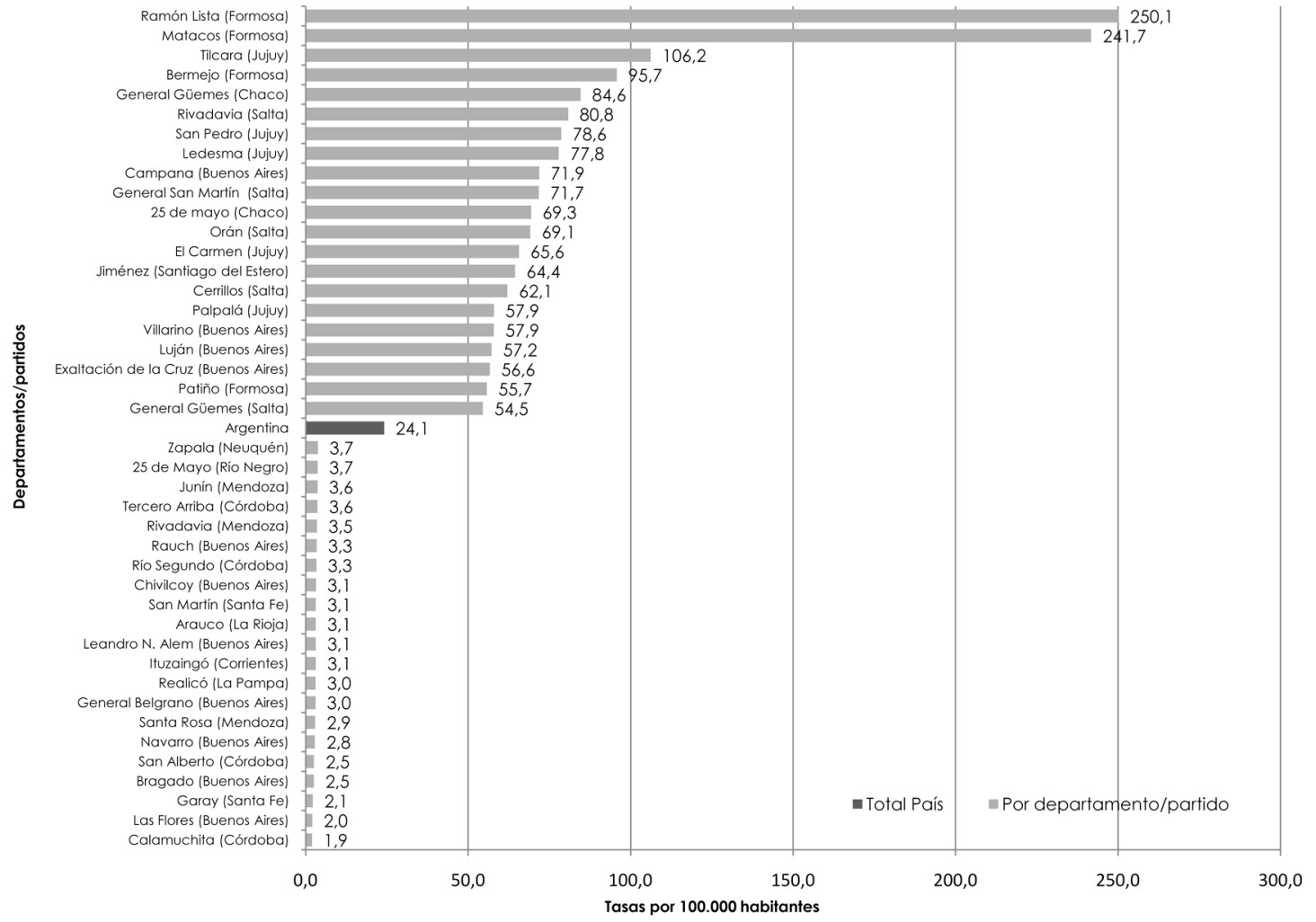

Fuente: Elaboración propia a partir de información oficial de los programas de control de la tuberculosis de las 24 jurisdicciones del país, consolidada por el Instituto Nacional de Enfermedades Respiratorias (INER) "Emilio Coni", Administración Nacional de Laboratorios e Institutos de Salud (ANLIS), del Ministerio de Salud de la Nación, Argentina, 2012.

2011. Esto contrasta con la situación de 149 departamentos que, también concentrando un $10 \%$ de la población del país, notificaron solo el $1,4 \%$ de todos los casos de la enfermedad.

También entre departamentos la desigualdad, medida mediante el índice de Gini, fue superior para las mujeres que para los hombres: 0,411 y 0,394 , respectivamente. En los varones, los 27 departamentos con mayor tasa que concentran el $10 \%$ de la población aportaron el 23,9\% de los casos y, en las mujeres, los 36 departamentos que concentran una misma proporción de población de mujeres, aportaron el 25,6\% de los casos de tuberculosis.

Las brechas en la distribución de la tuberculosis también se observan en las áreas urbanas en las cuales la probabilidad de diseminación de la enfermedad es mucho mayor que en los departamentos con poblaciones dispersas.
Analizando la situación de los 24 partidos del Gran Buenos Aires, en los que residían 3.257 de los 4.423 casos notificados en la provincia de Buenos Aires (73,6\%), se puede observar que la tasa de notificación en La Matanza fue 47,41 por 100.000, cifra tres veces mayor a la registrada en Hurlingham (15,51 por 100.000). La medida de la desigualdad, expresada a través del índice de Gini, fue de 0,148.

La desigualdad en la distribución del problema entre los partidos del Gran Buenos Aires fue mayor si se consideran solamente los casos de tuberculosis en jóvenes ya que la tasa de notificación de 20 a 34 años en La Matanza fue 69,07 por 100.000 , cifra cuatro veces superior a la de Ituzaingó (16,41 por 100.000 habitantes). En este caso, el índice de Gini fue superior: 0,204 . La desigualdad, a su vez, fue mayor en mujeres que en varones. 
Las tasas de notificación de casos en mujeres de 20 a 34 años fueron 6,4 veces superiores en La Matanza que en Ituzaingó: 63,99 y 10,00 por 100.000 , respectivamente. El índice de Gini para medir la desigual distribución de la tuberculosis en mujeres entre los partidos del Gran Buenos Aires fue 0,225, un $12 \%$ más alto que el de los hombres: 0,201. La brecha entre partidos para la tasa de notificación de casos en varones jóvenes de 20 a 34 años, fue considerablemente menor que en las mujeres: la tasa de La Matanza (74,24 por 100.000) fue 3,6 veces superior a la de Morón, con la menor tasa de notificación en varones: 20,41 por 100.000.

\section{Desigualdad social}

La distribución desigual de la tuberculosis en Argentina se asoció a las condiciones sociales y los departamentos con indicadores sociales más desfavorables tuvieron una contribución proporcionalmente mayor a la notificación de casos con relación a la contribución proporcional de su población.
Los 155 departamentos con mayor proporción de población que habitaban en viviendas precarias en 2010 (7,17\% o más de su población), que concentran un $20 \%$ del total de la población del país, aportaron un 29,1\% del total de casos de tuberculosis notificado en el bienio 2010-2011. En contraste, los 113 departamentos con menor proporción de población que habitaban en viviendas precarias en 2010 (menos del $1,62 \%$ de su población) y que también concentraron un $20 \%$ de la población total del país, contribuyeron con el $8,4 \%$ de todos los casos de tuberculosis notificados. El índice de concentración, calculado para la distribución de casos de tuberculosis en función de la proporción de población en viviendas precarias, fue -0,212.

Analizando la desigualdad en la distribución de la tuberculosis en varones y en mujeres, se observó una mayor asociación entre las condiciones desfavorables de vida de las mujeres que entre los hombres. Al utilizar la proporción de población de diez años o más analfabeta en varones y en mujeres, y relacionar este indicador con la tasa de notificación de casos de tuberculosis por departamento, se observó que, en las mujeres, el índice de

Figura 4. Tendencia temporal de la notificación de casos de tuberculosis (todas las formas y pulmonares examen directo positivo). Tasas (por 100.000 habitantes). Argentina, 1990-2011.

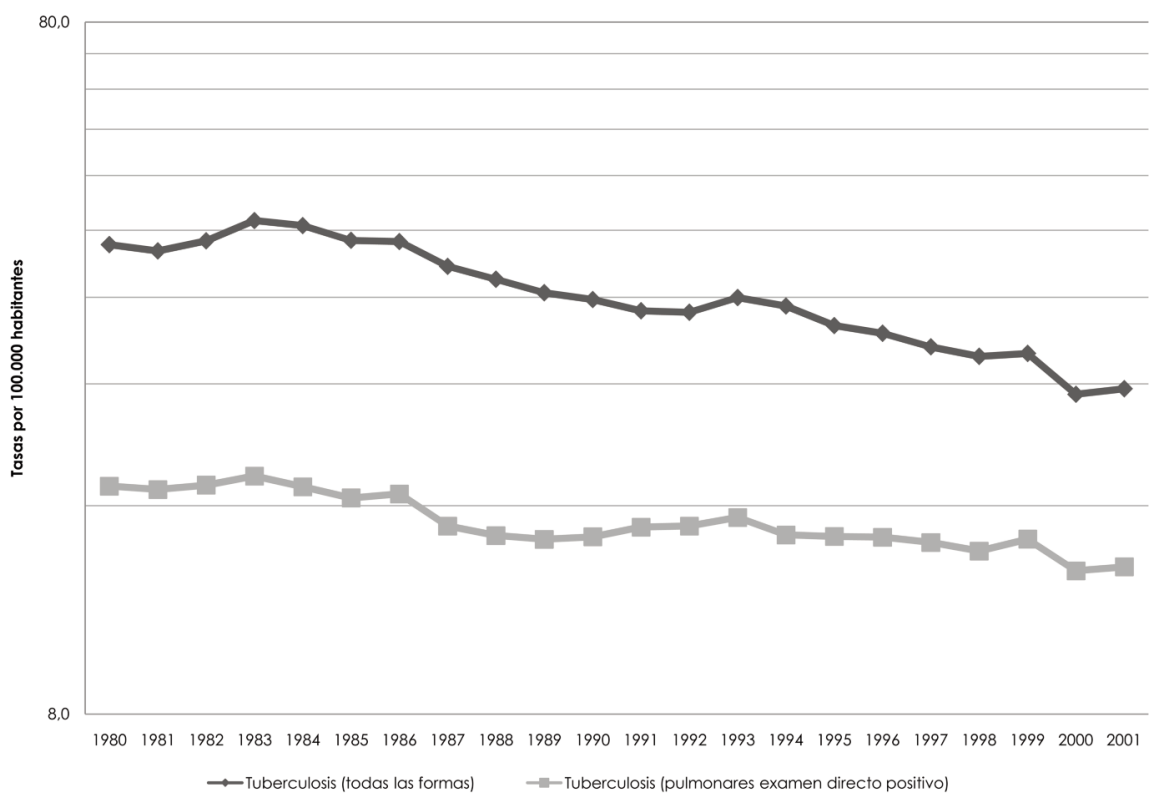

Fuente: Elaboración propia a partir de información oficial de los programas de control de la tuberculosis de las 24 jurisdicciones del país, consolidada por el Instituto Nacional de Enfermedades Respiratorias (INER) "Emilio Coni", Administración Nacional de Laboratorios e Institutos de Salud (ANLIS), del Ministerio de Salud de la Nación, Argentina, 2012. 
concentración fue de -0,108, mientras que no se observó asociación para los varones, con un índice de concentración de $+0,044$.

Un resultado equivalente se obtuvo analizando la relación entre la tasa de notificación de casos de tuberculosis y el porcentaje de población de 10 años o más analfabeta mediante regresión. Por cada incremento de un punto en el porcentaje de mujeres de 10 años o más analfabetas, los departamentos tuvieron un incremento de 11 puntos en la tasa de notificación de casos de tuberculosis, con un intervalo de confianza del $95 \%$ que fue de 6,4 a 16,4 (coeficiente de

Cuadro 5. Tendencia temporal de la notificación de casos de tuberculosis (todas las formas y pulmonares examen directo positivo). Números y tasas (por 100.000 habitantes). Argentina, 1990-2011.

\begin{tabular}{|c|c|c|c|c|}
\hline \multirow[t]{2}{*}{ Años } & \multicolumn{2}{|c|}{$\begin{array}{c}\text { Tuberculosis } \\
\text { (todas las formas) }\end{array}$} & \multicolumn{2}{|c|}{$\begin{array}{l}\text { Tuberculosis (pulmonare } \\
\text { examen directo positivo) }\end{array}$} \\
\hline & No. & Tasa & No. & Tasa \\
\hline 1990 & 12.355 & 38,1 & 5.533 & 17,1 \\
\hline 1991 & 12.181 & 37,4 & 5.508 & 16,9 \\
\hline 1992 & 12.596 & 38,6 & 5.587 & 17,1 \\
\hline 1993 & 13.914 & 41,3 & 5.948 & 17,7 \\
\hline 1994 & 13.683 & 40,6 & 5.738 & 17,0 \\
\hline 1995 & 13.450 & 38,7 & 5.707 & 16,4 \\
\hline 1996 & 13.397 & 38,5 & 5.787 & 16,6 \\
\hline 1997 & 12.621 & 35,5 & 5.319 & 15,0 \\
\hline 1998 & 12.276 & 34,0 & 5.234 & 14,5 \\
\hline 1999 & 11.871 & 32,5 & 5.234 & 14,3 \\
\hline 2000 & 11.767 & 31,8 & 5.341 & 14,4 \\
\hline 2001 & 11.464 & 30,6 & 5.595 & 14,9 \\
\hline 2002 & 11.545 & 30,5 & 5.668 & 15,0 \\
\hline 2003 & 12.278 & 32,0 & 5.910 & 15,4 \\
\hline 2004 & 12.079 & 31,1 & 5.642 & 14,5 \\
\hline 2005 & 11.242 & 29,1 & 5.575 & 14,5 \\
\hline 2006 & 11.068 & 28,4 & 5.616 & 14,4 \\
\hline 2007 & 10.683 & 27,1 & 5.574 & 14,2 \\
\hline 2008 & 10.452 & 26,3 & 5.469 & 13,8 \\
\hline 2009 & 10.657 & 26,6 & 5.745 & 14,3 \\
\hline 2010 & 9.393 & 23,2 & 5.221 & 12,9 \\
\hline 2011 & 9.657 & 23,6 & 5.338 & 13,1 \\
\hline
\end{tabular}

Fuente: Elaboración propia a partir de información oficial de los programas de control de la tuberculosis de las 24 jurisdicciones del país, consolidada por el Instituto Nacional de Enfermedades Respiratorias (INER) "Emilio Coni", Administración Nacional de Laboratorios e Institutos de Salud (ANLIS), del Ministerio de Salud de la Nación, Argentina, 2012. correlación de 0,75 , con un intervalo de confianza del 95\% de 0,46 a 0,90). El mismo análisis realizado para los varones no arrojó resultados estadísticamente significativos (coeficiente de correlación de 0,22 , con un intervalo de confianza de $-0,24$ a $+0,61)$.

La relación entre la mayor notificación de casos de tuberculosis y las condiciones sociales más desfavorables también se observó al analizar las áreas urbanas con mayor concentración de población. Entre los 24 partidos del Gran Buenos Aires, el índice de concentración varió entre -0,083 para la asociación entre la mayor notificación de casos de tuberculosis y la proporción de población en viviendas sin baño, y - 0,116 para la asociación con la proporción de población de diez años o más analfabeta.

Al igual que lo observado con la desigualdad, que fue mayor para la notificación de casos de tuberculosis entre jóvenes que en todas las edades, también se observó una mayor asociación con las condiciones sociales desfavorables. El índice de concentración para la tasa de notificación de casos de tuberculosis pulmonar por examen directo positivo de 20 a 34 años según la proporción de población de diez años o más analfabeta fue -0,162 y según la proporción de población en viviendas precarias fue de $-0,139$, cifras $39 \%$ más altas que las observadas para el total de los casos de tuberculosis de todas las edades.

La asociación entre la notificación de casos de tuberculosis y las condiciones sociales fue también más fuerte en las mujeres que en los varones. El índice de concentración para las mujeres fue de $-0,182$, un $22 \%$ más alto que en varones $(-0,149)$.

\section{Evolución del problema y de la desigualdad}

La tuberculosis se redujo en el país en los últimos decenios (Cuadro 5, Figura 4). El número de casos de todas las formas de la enfermedad se redujo 21,8\% entre 1990 y 2011 y la tasa de notificación disminuyó en el mismo período $38,1 \%$. Sin embargo, la reducción fue menor para los casos pulmonares confirmados por examen directo: el número de casos disminuyó solo 3,5\% y la tasa se redujo 23,6\%.

El descenso en la notificación de casos fue más rápido durante el último período (2000- 
2011), aunque la diferencia en la variación porcentual anual promedio con el período 1990-2000 no fue estadísticamente significativa (Cuadro 6). La velocidad de descenso de la notificación de casos pulmonares confirmados por examen directo fue menor en ambos períodos y también se observó una mayor reducción promedio durante el período 2000-2011 pero tampoco en este caso la diferencia fue estadísticamente significativa.

Cuadro 6. Variación porcentual anual promedio (e intervalos de confianza del 95\%) de la tendencia temporal de la notificación de casos de tuberculosis. Argentina, 1990-2011.

\begin{tabular}{|c|c|c|}
\hline Jurisdicción & $\begin{array}{l}\text { Variación anual } \\
\text { promedio (\%) }\end{array}$ & IC95\% \\
\hline Buenos Aires & $-2,0$ & $(-2,4 ;-1,5)$ \\
\hline CABA & 1,9 & $(1,2 ; 2,7)$ \\
\hline Catamarca & $-3,6$ & $(-5,2 ;-1,9)$ \\
\hline Córdoba & $-3,5$ & $(-4,1 ;-2,9)$ \\
\hline Corrientes & $-2,2$ & $(-3,3 ;-1,2)$ \\
\hline Chaco & $-1,6$ & $(-2,7 ;-0,4)$ \\
\hline Chubut & $-4,4$ & $(-5,2 ;-3,7)$ \\
\hline Entre Ríos & $-5,0$ & $(-6,1 ;-3,9)$ \\
\hline Formosa & $-3,6$ & $(-4,4 ;-2,7)$ \\
\hline Jujuy & $-4,9$ & $(-5,7 ;-4,2)$ \\
\hline La Pampa & $-3,6$ & $(-4,9 ;-2,3)$ \\
\hline La Rioja & $-8,2$ & $(-9,3 ;-7,0)$ \\
\hline Mendoza & $-4,9$ & $(-6,0 ;-3,8)$ \\
\hline Misiones & $-3,3$ & $(-4,4 ;-2,3)$ \\
\hline Neuquén & $-8,8$ & $(-10,3 ;-7,3)$ \\
\hline Río Negro & $-2,7$ & $(-5,4 ; 0,1)$ \\
\hline Salta & $-4,3$ & $(-4,9 ;-3,7)$ \\
\hline San Juan & $-5,0$ & $(-6,5 ;-3,4)$ \\
\hline San Luis & $-4,6$ & $(-6,8 ;-2,4)$ \\
\hline Santa Cruz & $-4,5$ & $(-5,2 ;-3,7)$ \\
\hline Santa Fe & $-3,1$ & $(-3,4 ;-2,7)$ \\
\hline Santiago del Estero & $-1,3$ & $(-2,7 ; 0,2)$ \\
\hline Tierra del Fuego & $-7,3$ & $(-9,2 ;-5,4)$ \\
\hline Tucumán & $-3,4$ & $(-4,4 ;-2,4)$ \\
\hline Total & $-2,5$ & $(-2,9 ;-2,2)$ \\
\hline
\end{tabular}

Fuente: Elaboración propia a partir de información oficial de los programas de control de la tuberculosis de las 24 jurisdicciones del país, consolidada por el Instituto Nacional de Enfermedades Respiratorias (INER) "Emilio Coni", Administración Nacional de Laboratorios e Institutos de Salud (ANLIS), del Ministerio de Salud de la Nación, Argentina, 2012

CABA = Ciudad Autónoma de Buenos Aires.
La tendencia de la notificación de casos de tuberculosis fue diferente en las 24 jurisdicciones (Cuadro 6). En la Ciudad Autónoma de Buenos Aires, la tasa de notificación de casos de tuberculosis (todas las formas) se incrementó 48,7\% entre 1990 y 2011, y tuvo una variación porcentual anual promedio al aumento de 1,9\% (entre 1,17\% y $2,65 \%$ ). En las 23 provincias la tendencia fue al descenso pero el ritmo de descenso fue diferente: en Santiago del Estero la tasa se redujo al 1,27\% anual y en Neuquén fue del 8,48\% anual.

La tendencia desigual que registraron las jurisdicciones entre 1990 y 2011 no contribuyó a reducir la desigualdad entre ellas. El índice de Gini se mantuvo con un valor similar: 0,253 en 1990 0,243 en 2000 y se incrementó a 0,273 en 2011.

La desigualdad en la distribución del total de casos de tuberculosis asociada a las condiciones sociales tampoco mostró variaciones entre 1990 y 2011: el índice de concentración tuvo el mismo valor en 1990 y $2000(0,122)$ y se incrementó a 0,136 en 2011.

\section{DISCUSIÓN Y CONCLUSIONES}

La tuberculosis en Argentina es un importante problema de salud pública, que cada año produce cerca de 10.000 casos registrados en el país, que representan una carga de enfermedad que afecta la salud de la población de manera significativa. Incluso es probable que la cantidad real de casos sea mayor, dadas las deficiencias y limitaciones que tienen los sistemas de registro y notificación de casos de enfermedades, que no incluyen todos los que realmente están ocurriendo y suelen tener un subregistro que puede ser variable, aunque en el caso de la tuberculosis en Argentina se publica que la diferencia entre los casos estimados y los registrados es relativamente pequeña.

Como comparación, en el contexto de otras enfermedades que son objeto de vigilancia epidemiológica y que cuentan con programas de control en Argentina, se notifican anualmente cerca de 1.300 nuevos casos de sida, poco más de 4.000 nuevas infecciones por $\mathrm{VIH}$, casi 700 casos de hepatitis B y entre 300 y 400 casos de lepra en el país, por mencionar solo algunas de 
las enfermedades trasmisibles crónicas $(17,18)$. Frente a los casi 10.000 casos nuevos registrados de tuberculosis, se destaca la importancia en cuanto a la magnitud de este problema de salud. Según la información que se dispone, la tuberculosis es el evento de vigilancia epidemiológica en el país que más casos nuevos produce cada año entre las patologías crónicas mencionadas, superior incluso a la suma de todas ellas juntas.

Es claro que los criterios de priorización para la atención de problemas de salud pública deben tener en cuenta la magnitud del problema, y desde esta perspectiva la tuberculosis es un problema que debe ser priorizado.

A su vez, también se destaca que la tuberculosis en Argentina es un problema de salud de importancia no solo por su magnitud sino que el análisis de las características de los grupos afectados por ella en nuestro país nos muestra el impacto especial que el problema tiene en la población más vulnerable (como las mujeres y la población con condiciones sociales desfavorables) y en la población joven (20 a 34 años).

El problema se distribuye de manera desigual, y las brechas son importantes en la población joven del país, donde la afectación especial en la población de 20 a 34 años representa un impacto importante en un grupo poblacional motor de la economía en la sociedad y su enfermedad puede afectar la dinámica familiar en muchos casos.

La desigualdad se repite en la distribución por provincias y las brechas son mucho mayores en el análisis por departamentos. Se observa que las provincias y departamentos con condiciones sociales y económicas más adversas tienen una carga de enfermedad mucho más alta que los que tienen mejores condiciones. Se destaca también el hallazgo de una concentración mayor del problema en las áreas altamente urbanizadas con grandes cantidades de población, como en el Gran Buenos Aires.

También, al analizar en detalle, se observa la desigualdad por género. Aunque la tuberculosis se presenta con mayor magnitud en hombres, al cruzar la información combinada de género, edad y condición social emerge la situación desigual de la distribución del problema en la mujer. Finalmente, y en relación con los elementos y características que se incluyeron en este análisis, se confirma en el presente estudio la asociación entre mayor concentración y producción de casos de tuberculosis y las malas condiciones sociales y económicas de la población.

La vulnerabilidad social de la población más afectada por la enfermedad, al igual que la magnitud mencionada anteriormente, es un criterio que también se tiene en cuenta para la priorización de los problemas de salud en cuanto a las acciones para su control.

La alta magnitud de un problema de salud que además es trasmisible por vía aérea, sumada a la afectación de grupos especiales de la población con alta movilidad y con alta vulnerabilidad, exponen el riesgo elevado que tiene la población más desfavorecida socialmente de verse afectada por un problema de salud que además es inhabilitante para el desarrollo y sostén económico y que alimenta el círculo de discriminación y estigmatización al que pueden estar sometidos estos grupos de población $(2,16)$.

A su vez, en virtud de la demostración de la desigualdad visible en los lugares de concentración urbana y en poblaciones con alta movilidad, las condiciones de la enfermedad y de las personas afectadas -quienes adicionalmente son la fuente de diseminación de la enfermedad- exponen además que, aunque la tuberculosis afecta más a la población vulnerable, toda la sociedad está sujeta al riesgo de desarrollar la enfermedad y este riesgo es mayor en la medida que existan más casos, independientemente de donde se produzcan.

La evolución del problema también merece consideración en virtud de que aun cuando la tuberculosis ha venido disminuyendo su magnitud en el período 1990-2011, el descenso no tiene el ritmo que garantice un logro importante en materia del control del problema y no es compatible con la reducción de la desigualdad en la distribución de la enfermedad. A pesar de contar con una reducción global de 2,5\% anual promedio para Argentina, algunos países de nuestro continente presentan reducciones de la incidencia mucho más importantes como Chile $(6,7 \%)$, Cuba $(8,0 \%)$ o EE.UU. (6,0\%), o incluso otros países de América Latina como Brasil presentan una reducción mayor que Argentina o a lo sumo similar (3,0\%) (1). 
El logro de una tendencia compatible con el control de la enfermedad -uno de los Objetivos del Milenio a los que Argentina adhirió- implica un aumento del ritmo de descenso de la tuberculosis pero, además, requiere un incremento mayor en el descenso del problema en los grupos en peor situación, de manera de reducir la desigualdad existente en la distribución de la tuberculosis, que no se observó en los últimos decenios $(6,15,19)$.

Se considera que el marco de importancia del problema, la inequidad en la afectación de la población y el ritmo lento de reducción que hay en nuestro país ubican a Argentina en la necesidad de reforzar las acciones de atención del problema, incluyendo la aplicación de las estrategias recomendadas para luchar contra la tuberculosis, fomentando cada vez más el involucramiento de la comunidad para analizar, planificar y resolver el problema.

Por último, nos parece importante mencionar que la profundización de los análisis de la abundante y diversa información disponible sobre notificación, resultado del tratamiento y mortalidad por tuberculosis son un camino necesario para comprender mejor el problema y, por ende, para prever mejor su solución.

\section{REFERENCIAS BIBLIOGRÁFICAS}

1. World Health Organization. Global tuberculosis control: surveillance, planning, financing. Geneva: WHO; 2011.

2. Alianza Alto a la Tuberculosis, Organización Mundial de la Salud. Plan Mundial para Detener la Tuberculosis 2006-2015 (WHO/ HTM/STB/2006,35). Ginebra: Organización Mundial de la Salud; 2006.

3. Dye C, Maher D, Weil D, Espinal M, Raviglione M. Targets for global tuberculosis control. International Journal of Tuberculosis and Lung Disease. 2006;10(4):460-462.

4. Glaziou P, Floyd K, Raviglione M. Global burden and epidemiology of tuberculosis. Clinic inChest Medicine. 2009;30(4):621-636.

5. Caminero-Luna JA. Guía de la tuberculosis para médicos especialistas. París: Unión Internacional Contra la Tuberculosis y Enfermedades Respiratorias (UICTER); 2003.

6. Lonnroth K, Castro KG, Chakaya JM, Chauhan LS, Floyd K, Glaziou P, Raviglione MC. Tuberculosis control and elimination 2010-50: cure, care, and social development. Lancet. 2010;375(9728):1814-1829.

7. Hargreaves JR, Boccia D, Evans CA, Adato M, Petticrew M, Porter JD. The social determinants of tuberculosis: from evidence to action. American Journal of Public Health. 2011;101(4):654-662.
8. Lonnroth K, Jaramillo E, Williams BG, Dye C, Raviglione MC. Drivers of tuberculosis epidemics: the role of risk factors and social determinants. Social Sciences \& Medicine. 2009;68(12):2240-2246.

9. Creswell J, Jaramillo E, Lonnroth K, Weil D, Raviglione M. Tuberculosis and poverty: what is being done. International Journal of Tuberculosis and Lung Disease. 2011;15(4):431-432.

10. Chaulet $\mathrm{P}$, Raviglione $\mathrm{M}$, Bustreo $\mathrm{F}$. Epidemiology, control and treatment of multidrug-resistant tuberculosis. Drugs. 1996;52(Suppl 2):S103-S107.

11. Palmero DJ, Laniado-Laborin R, CamineroLuna JA. Latin American guidelines for the diagnosis and management of drug-resistant tuberculosis. Archivos de Bronconeumología. 2008;44(10):578.

12. Murray M, Oxlade $\mathrm{O}$, Lin $\mathrm{HH}$. Modeling social, environmental and biological determinants of tuberculosis. International Journal of Tuberculosis and Lung Disease. 2011;15(Suppl 2):S64-S70.

13. Rasanathan K, Sivasankara-Kurup A, Jaramillo $E$, Lönnroth $K$. The social determinants of health: key to global tuberculosis control. International Journal of Tuberculosis and Lung Disease. 2011;15(Suppl 2):S30-S36.

14. Bossio J, Arias S, Veronesi I, López R, Colombini R. Notificación de casos de tuberculosis en la República Argentina: Período 1980-2010. Santa Fe: Departamento Programas de Salud, INER "Emilio Coni", ANLIS "Carlos G. Malbrán"; 2011. 
15. Dye C, Lonnroth K, Jaramillo E, Williams BG, Raviglione M. Trends in tuberculosis incidence and their determinants in 134 countries. Bulletin of the World Health Organization. 2009;87(9):683-691.

16. Lönnroth K, Raviglione M. Global epidemiology of tuberculosis: prospects for control. Seminars in Respiratory and Critical Care Medicine. 2008;29(5):481-491.

17. Secretaría de Promoción y Programas Sanitarios. Boletín Integrado de Vigilancia $\mathrm{N}^{\circ}$ 107 [Internet]. Buenos Aires: Ministerio de Salud; 2012 [citado 20 sep 2012]. Disponible en: http://www.msal.gov.ar/images/stories/boleti-
nes/BoletinlntegradoDeVigilanciaVersion N107SE05.pdf.

18. Sociedad Argentina de Dermatología. XIII Conferederal de lepra de la República Argentina: enfermos de lepra de Argentina al 31/12/2011 [Internet] 2012 [citado 12 sep 2012]. Disponible en: http://www.sad.org.ar/lepra/docs/TABLAXIII31-12-2011.pdf

19. Guimarães RM, Lobo AD, Siqueira EA, Borges TF, Melo SC. Tuberculosis, HIV, and poverty: temporal trends in Brazil, the Americas, and worldwide. Jornal Brasileiro de Pneumologia. 2012;38(4):511-517.

\section{FORMA DE CITAR}

Bossio JC, Arias SJ, Fernández HR. Tuberculosis en Argentina: desigualdad social y de género. Salud Colectiva. 2012;8(Supl 1):S77-S91.

Recibido el 15 de septiembre de 2012

Aprobado el 9 de octubre de 2012 